\title{
On the Stability of Quartic Functional Equations via Fixed Point and Direct Method
}

\author{
Renu Chugh ${ }^{1}$, Ashish $^{2}$, Manoj Kumar ${ }^{3}$ \\ ${ }^{1}$ Professor, ${ }^{2}$ Research Scholar, ${ }^{3}$ Assistant Professor \\ Dept. of Mathematics \\ M. D. University \\ Rohtak-124001, INDIA
}

\begin{abstract}
The purpose of this paper is to establish the Hyers-UlamRassias stability of quartic functional equation $f(3 x+y)+f(x+3 y)=64 f(x)+64 f(y)+24 f(x+y)-6 f(x-y)$ in the setting of random normed space and intuitionistic random normed space. The stability of the equation is proved by using the fixed point method and direct method.
\end{abstract}

\section{Keywords}

Fixed point method, Quartic functional equation, Random normed space, intuitionistic random normed space

\section{INTRODUCTION AND PRELIMINARIES}

One of the interesting questions in the theory of non-linear functional analysis involved is the stability problem of functional equations as follows: "When is it true that a mathematical object satisfying a certain property approximately must be close to an object satisfying the property exactly?" The stability problem of functional equations was first raised by S. M. Ulam [16], concerning the stability of group homomorphism in 1940:

Let $\left(G_{1}, *\right)$ be a group and let $\left(G_{2}, \diamond, d\right)$ be a metric group with the metric $d$ (., .). Given $\varepsilon>0$, does there exists a $\delta(\varepsilon)>0$ such that if a mapping $h: G_{l} \rightarrow G_{2}$ satisfies the following inequality $d\left(h\left(x^{*} y\right), h(x) \diamond h(y)\right)<\delta$, for all $x, y \in G_{l}$, then there is a homomorphism $H: G_{l} \rightarrow$ $G_{2}$ with $\quad d(h(x), H(x))<\varepsilon$, for all $x \in G_{1}$ ?

If the answer is affirmative, we would say that equation of homomorphism $H(x y)=H(x) H(y)$ is stable.

In the next year, D. H. Hyer [4] gave the first affirmative answer of the Ulam's problem for additive mapping $f(x+y)=f(x)+f(y)$ on Banach spaces. A generalized version of the theorem of Hyers [4] was given by Th. M. Rassias [17] in 1978 which allows Cauchy difference to be unbounded. The generalization given by Th. M. Rassias [17] is called the Hyers-Ulam-Rassias stability. In1994, P. Gavruta [11] provided a further generalization of Th. M. Rassias [17] theorem in which he replaced the bound $\varepsilon\left(\|x\|^{p}+\|y\|^{p}\right)$ by a general function $\phi(x, y)$ for the existence of unique linear mapping. The Hyers-Ulam-Rassias stability of various functional equations have been extensively introduced by a number of Mathematicians.The functional equation

$f(3 x+y)+f(x+3 y)=64 f(x)+64 f(y)+24 f(x+y)-6 f(x-y)$

for all $x, y \in X$, is called the Quartic functional equation, since $a x^{4}$ is a solution of the Quartic functional equation (1.1). The Hyers-Ulam-Rassias stability of this equation was introduced by M. Petapirak and P. Nakmahachalasint [10] in 2008 for the mapping $f: X \rightarrow Y$, where $X$ is a real normed space and $Y$ is a real Banach space.

We organize this paper in four sections as follows: In section 1, we study the introduction and preliminaries related to our results. The Hyers-UlamRassias stability of the quartic functional equation (1.1) using fixed point method on random normed space is given in section 2. In Section 3 using the direct approach, we prove the stability of the quartic functional equation (1.1) in intuitionistic random normed space. In the last section, we gave the concluded remarks of the paper.

Now, first we study some definitions, results and notations for the next sections which have been already studied. (see [1], [2], [3], [5], [6]) 
Definition 1.2 [2] A mapping $T:[0,1] \times[0,1] \rightarrow[0,1]$ is a continuous triangular norm (briefly a $t$ - norm) if $T$ satisfies the following conditions :
(a) $\quad T$ is commutative and associative;
(b) $T$ is continuous;
(c) $\quad T(a, 1)=a$ for all $a \in[0,1]$;
(d) $\quad T(a, b) \leq T(c, d)$ whenever $a \leq c$ and $b \leq d$ for all
$a, \quad b, c, d \in[0,1]$.

Typical examples of continuous $t$-norm are $T(a, b)=a b, T$ $(a, b)=\max (a+b-1,0)$ and $T(a, b)=\min (a, b)$

Definition 1.2 [1] A Random Normed space (briefly RNspace $)$ is a triple $(X, \mu, T)$, where $X$ is a vector space, $T$ is a continuous $t$-norm, and $\mu$ is a mapping from $X$ into $D^{+}$ such that the following conditions hold:

(RN1) $\mu_{x}(t)=\varepsilon_{0}(t)$ for all $t>0$ if and only if $x=0$;

(RN2) $\mu_{\alpha x}(t)=\mu_{x}(t /|\alpha|)$ for all $x$ in $X, \alpha \neq 0$ and all $t \geq 0$;

(RN3) $\mu_{x+y}(t+s) \geq T\left(\mu_{x}(t), \mu_{y}(s)\right)$ for all $x$, $y \in X$ and all $t, s \geq 0$

Definition 1.3 Let $(X, \mu, T)$ be an $\mathrm{RN}$ - space

(1) A sequence $\left\{x_{n}\right\}$ in $X$ is said to be convergent to $x$ in $X$ if, for every $t>0$ and $\varepsilon>0$, there exists a positive integer $N$ such that $\mu_{x_{n}-x}(t)>1-\varepsilon$ whenever $n \geq N$.

(2) A sequence $\left\{x_{n}\right\}$ in $X$ is said to be Cauchy sequence if, for every $t>0$ and $\varepsilon>0$, there exists a positive integer $N$ such that $\mu_{x_{n}-x_{m}}(t)>1-\varepsilon$ whenever $n \geq m \geq N$.

(3) An RN- space $(X, \mu, T)$ is said to be complete if and only if every Cauchy sequence in $X$ is convergent to a point in $X$.

Theorem 1.4 [2] If $(X, \mu, T)$ is an RN-space and $\left\{x_{n}\right\}$ is a sequence such that $x_{n} \rightarrow x$, then $\operatorname{Lim}_{n \rightarrow \infty} \mu_{x_{n}}(t)=\mu_{x}(t)$ almost everywhere.

Theorem 1.5 (Fixed point alternative). Let $(X, d)$ be a complete generalized metric space and a contractive mapping $J: X \rightarrow X$, with the Lipschitz constant $L$. Then, for each given element $x \in X$, either

$\left(\mathrm{A}_{1}\right) \quad d\left(J^{n} x, J^{n+1} x\right)=+\infty$ for all $n \geq 0$,

Or
$\left(\mathrm{A}_{2}\right)$ There exists a natural $n_{0}$ such that:

$\left(\mathrm{A}_{20}\right) \quad d\left(J^{n} x, J^{n+1} x\right)=+\infty$ for all $n \geq n_{0}$,

$\left(\mathrm{A}_{21}\right)$ The sequence $\left(J^{n} x\right)$ is convergent to a fixed point $y^{*}$ of $J$

$\left(\mathrm{A}_{22}\right) y^{*}$ is the unique fixed point of $J$ in the set $Y=\left\{y \in X, d\left(J^{n_{O}}, y\right)<+\infty\right\}$;

$\left(\mathrm{A}_{23}\right) \quad d\left(y, y^{*}\right) \leq \frac{1}{1-L} d(y, J y)$, for all $y \in Y$.

The following lemmas will be used in the proof of theorem (2.1)

Lemma 1.6 [3] Let $(X, d)$ be a complete generalized metric space and let $A: X \rightarrow X$ be a strict contraction with the Lipschitz constant L such that $d\left(x_{0}, A\left(x_{0}\right)\right)<+\infty$ for some $x_{0} \in X$. Then $A$ has a unique fixed point in the set $Y=$ $\left\{y \in X, d\left(x_{0}, y\right)<\infty\right\}$ and the sequence $\left(A^{\mathrm{n}}(x)\right)_{\mathrm{n} \in \mathrm{N}}$ converges to the fixed point $x^{*}$ for every $x \in Y$. Moreover, $d\left(x_{0}, A\left(x_{0}\right)\right) \leq \delta$ implies $d\left(x^{*}, x_{0}\right) \leq \delta / 1-L$.

Lemma $1.7[5,6] d_{\mathrm{G}}$ is a complete generalized metric on E.

\section{RANDOM NORMED STABILITY OF THE QUARTIC FUNCTIONAL EQUATIONS USING FIXED POINT METHOD}

In this section, we shall prove the Hyers-UlamRassias stability of quartic functional equation (1.1) in random normed space using fixed point method.

Throughout, this section, we consider $X$ as a linear space and $Y$ as a complete random normed space.

Theorem 2.1 Let $X$ be a real linear space, ' $f$ ' be a mapping from $X$ into a complete random normed space $\left(Y, \mu, T_{M}\right)$ with $f(0)=0$ and let $\Phi: X \times X \rightarrow D^{+}$be a mapping

satisfying

$\mu_{(f(3 x+y)+f(x+3 y)-64 f(x)-64 f(y)-24 f(x+y)+6 f(x-y))}(t) \geq \Phi_{x, y}(t)$

(2.2)

for all $x, y \in X$ then there exists a unique mapping $g: X \rightarrow Y$ such that $\mu_{f(x)-g(x)}(t) \geq \Phi_{x, 0}\left(\left(3^{4}-\alpha\right) t\right)$ for all $x \in X$ and all $t>0$.

Proof: Taking $y=0$ in (2.2), we get

$$
\mu_{\left(f(3 x)-3^{4} f(x)\right)}(t) \geq \Phi_{x, 0}(t) \text { for all } x \in X
$$

therefore 


$$
\begin{aligned}
\mu_{\left(\frac{1}{3^{4}} f(3 x)-f(x)\right)}(t)=\mu_{\frac{1}{3^{4}}\left(f(3 x)-3^{4} f(x)\right)}(t) \\
=\mu_{\left(f(3 x)-3^{4} f(x)\right)}\left(3^{4} t\right) \\
\geq \Phi_{x, 0}\left(3^{4} t\right) \text { for all } x \in X \text { and } t>0
\end{aligned}
$$

Now, to prove the stability using fixed point approach, let us consider a set $S=\{g: X \rightarrow Y\}$ and the mapping $d_{G}$ in $S$ defined by

$d_{G}(f, g)=\inf \left\{u \in R^{+}: \mu_{g(x)-h(x)}(u t) \geq \Phi_{x, 0}(t), \forall x \in X, t>0\right\}$

Now using Lemma 1.7, $\left(d_{G}, S\right)$ is a complete generalized metric space. Let us consider the linear mapping $J: S \rightarrow S$ such that

$$
J g(x)=\frac{g(3 x)}{3^{4}}
$$

We claim that the mapping $J$ is a strictly contractive self mapping of $S$ with the Lipschitz constant $\alpha / 3^{4}$. Let $g$ and $h$ be two mappings in $S$ such that $d_{G}(g, h)<\varepsilon$, then

$\mu_{g(x)-h(x)}(\varepsilon t) \geq \Phi_{x, 0}\left(3^{4} t\right) \quad$ for all $x \in X$ and $t>0$

Hence $\mu_{J g(x)-J h(x)}\left(\frac{\alpha}{3^{4}} \varepsilon t\right)=\mu_{\frac{1}{3^{4}}(g(3 x)-h(3 x))}\left(\frac{\alpha}{3^{4}} \varepsilon t\right)$

$$
\begin{aligned}
& =\mu_{(g(3 x)-h(3 x))}(\alpha \varepsilon t) \\
& \geq \Phi_{3 x, 0}\left(3^{4} \alpha t\right) \text { for all } x \in X, t>0
\end{aligned}
$$

Since $\quad \Phi_{3 x, 0}\left(3^{4} \alpha t\right) \geq \Phi_{x, 0}\left(3^{4} t\right), \mu_{J g(x)-J h(x)}\left(\frac{\alpha}{3^{4}} \varepsilon t\right)$ $\geq \Phi_{x, 0}\left(3^{4} t\right)$, that is

$$
d_{G}(g, h)<\varepsilon \Rightarrow d_{G}(J g, J h)<\frac{\alpha}{3^{4}} \varepsilon
$$

which implies that $d_{G}(J g, J h)<\frac{\alpha}{3^{4}} d_{G}(g, h)$ for all $g, h$ in S. Now, it follows from (2.5) that $d_{G}(f, J f) \leq 1$. Using the Lemma 1.6, we show the existence of a fixed point of $J$, that is the existence of a mapping $g: X \rightarrow Y$ satisfying the following:

$g$ is a fixed point of $J$, that is $g(3 x)=3^{4} g(x)$ for all $x$ in $X$.
Since for any $x$ in $X$ and $t>0, \quad d_{G}\left(J^{n} f, g\right)<\varepsilon$ implies $\quad \mu_{u(x)-v(x)}(t) \geq \Phi_{x, 0}\left(\frac{3^{4} t}{\varepsilon}\right), \quad$ from $d_{G}\left(J^{n} f, g\right) \rightarrow 0, \quad$ it follows that the $\lim _{n \rightarrow \infty} \frac{f\left(3^{n} x\right)}{3^{4 n}}=g(x)$ for all $x$ in $X$.

(iii) Also, $d_{G}(f, g) \leq \frac{1}{1-L} d_{G}(f, J f)$ implies that the inequality

$$
d_{G}(f, g) \leq \frac{1}{1-\frac{\alpha}{3^{4}}} d_{G}(f, J f)
$$

and so,

$$
\mu_{f(x)-g(x)}\left(\frac{3^{4} t}{3^{4}-\alpha}\right) \geq \Phi_{x, 0}\left(3^{4} t\right)
$$

for all $t>0$ and for all $x \in X$. It follows that $\mu_{f(x)-g(x)}(t) \geq \Phi_{x, 0}\left(\left(3^{4}-\alpha\right) t\right)$ for all $x \in X$ and all $t>0$.

The mapping $g$ is also unique, it follows from the fact that $g$ is the unique fixed point of $J$ with the property that, if there is a $T \in] 0, \infty[$ such that

$$
\mu_{f(x)-g(x)}(T t) \geq \Phi_{x, 0}\left(3^{4} t\right)
$$

for all $x \in X$ and for all $t>0$. This completes the proof of theorem.

\section{INTUITIONISTIC RANDOM NORMED STABILITY OF QUARTIC FUNCTIONAL EQUATION (1.1) USING DIRECT METHOD}

In this section, we prove the Hyers-Ulam-Rassias stability of the quartic functional equation (1.1) in intuitionistic random normed space.

First we present some definitions, results and conventions of theory of IRN spaces related to our results. (see $[1,2,5,8,12,13,14,15])$

Definition 3.1 A measure distribution function is a function $\mu: R \rightarrow[0,1]$ which is left continuous, nondecreasing on $R, \inf _{t \in R} \mu(t)=0$ and $\sup _{t \in R} \mu(t)=1$.

We will denote by $D$ the family of all measure distribution functions and by $H$ a special element of $D$ defined by

$$
H(t)=\left\{\begin{array}{lll}
0 & \text { if } & t \leq 0 \\
1 & \text { if } & t>0
\end{array}\right.
$$

If $X$ is a nonempty set, then $\mu: X \rightarrow D$ is called a probabilistic measure on $X$ and $\mu(x)$ is denoted by $\mu_{\mathrm{x}}$. 
Definition 3.2. A non-measure distribution function is a function $v: R \rightarrow[0,1]$ which is right continuous, nondecreasing on $R, \inf _{t \in R} v(t)=0$ and $\sup _{t \in R} v(t)=1$.

We will denote by $B$ the family of all non-measure distribution functions and by $G$ a special element of $B$ defined by

$$
G(t)=\left\{\begin{array}{lll}
1 & \text { if } & t \leq 0 \\
0 & \text { if } & t>0
\end{array}\right.
$$

If $X$ is a nonempty set, then $v: X \rightarrow B$ is called a probabilistic non-measure on $X$ and $v(x)$ is denoted by $v_{\mathrm{x}}$.

Lemma $3.3[7,9]$ Consider the set $L^{*}$ and operation $\leq_{L^{*}}$ defined by:

$L^{*}=\left\{\left(x_{1}, x_{2}\right):\left(x_{1}, x_{2}\right) \in[0,1]^{2}\right.$ and,$\left.x_{1}+x_{2} \leq 1\right\}$,

$\left(x_{1}, x_{2}\right) \leq_{L^{*}}\left(y_{1}, y_{2}\right) \Leftrightarrow x_{1} \leq y_{1}, x_{2} \geq y_{2}$,

$$
\forall\left(x_{1}, x_{2}\right),\left(y_{1}, y_{2}\right) \in L^{*}
$$

Then $\left(L^{*}, \leq_{L^{*}}\right)$ is a complete lattice.

We denote its units by $0_{L^{*}}=(0,1)$ and $1_{L^{*}}=(1,0)$. In section 1 , we presented classical $t$-norm. Using the lattice $\left(L^{*}, \leq_{L^{*}}\right)$, these definitions can be straightforwardly extended

Definition 3.4 [7] A triangular norm ( $t$-norm) on $L^{*}$ is a mapping $T:\left(L^{*}\right)^{2} \rightarrow L^{*}$ satisfying the following conditions:

(a) $\left(\forall x \in L^{*}\right)\left(T\left(x, 1_{L^{*}}\right)=x\right)$ (boundary condition);

(b) $\left(\forall(x, y) \in\left(L^{*}\right)^{2}\right)(T(x, y)=T(y, x))$ (commutativity);

(c) $\left(\forall(x, y, z) \in\left(L^{*}\right)^{3}\right)(T(x, T(y, z))=T(T(x, y), z))$

(associativity);

(d) $\left(\forall\left(x, x^{1}, y, y^{1}\right) \in\left(L^{*}\right)^{4}\right)\left(x \leq_{L^{\prime}} x^{1}\right.$ and,$\left.y \leq_{L^{*}} y^{1} \Rightarrow T(x, y) \leq_{L^{\prime}} T\left(x^{1}, y^{1}\right)\right)$

(monotonicity)

If $\left(L^{*}, \leq_{L^{*}}, T\right)$ is an abelian topological monoid with unit $1_{L^{*}}$ then $T$ is said to be a continuous $t$-norm.

Definition 3.5 [7] A continuous $t$-norm $T$ on $L^{*}$ is said to be continuous $t$-representable if there exist a continuous $t$ norm * and a continuous $t$-conorm $\diamond$ on $[0,1]$ such that, for all

$x=\left(x_{1}, x_{2}\right), y=\left(y_{1}, y_{2}\right) \in L^{*}, T(x, y)=\left(x_{1} * y_{1}, x_{2} \diamond y_{2}\right)$.

For example,

$$
T(a, b)=\left(a_{1} b_{1}, \min \left\{a_{2}+b_{2}, 1\right\}\right)
$$

and

$$
M(a, b)=\left(\min \left\{a_{1}, b_{1}\right\}, \max \left\{a_{2}, b_{2}\right\}\right)
$$

are continuous $t$-representable for all $a=\left(a_{1}, a_{2}\right), b=$ $\left(b_{1}, b_{2}\right) \in L^{*}$. Now, we define a sequence $T^{n}$ recursively by $T^{1}=T$ and

$T^{n}\left(x^{(1)}, \ldots, x^{(n+1)}\right)=T\left(T^{n-1}\left(x^{(1)}, \ldots, x^{(n)}\right), x^{(n+1)}\right), \forall n \geq 2$, $x^{(i)} \in L^{*}$.

Definition 3.6 A negator on $L^{*}$ is any decreasing mapping $N: L^{*} \rightarrow L^{*}$ satisfying $N\left(1_{L^{*}}\right)=0_{L^{*}}$ and $N\left(0_{L^{*}}\right)=1_{L^{*}}$. If $N(N(x))=x$ for all $\mathrm{x} \in L^{*}$, then $N$ is called an involutive negator. A negator on $[0,1]$ is a decreasing function $N$ : $[0$, $1] \rightarrow[0,1]$ satisfying $N(0)=1$ and $N(1)=0 . N_{s}$ denotes the standard negator on $[0,1]$ defined by $N_{s}(x)=1-x, \forall$ $x \in[0,1]$.

Definition 3.7 Let $\mu$ and $v$ be measure and non-measure distribution functions from $X \times(0,+\infty)$ to $[0,1]$ such that $\mu_{x}(t)+v_{x}(t) \leq 1$ for all $x \in X$ and $t>0$. The triple $\left(X, P_{\mu, v}, T\right)$ is said to be an intuitionistic random normed space (briefly IRN-space) if $\mathrm{X}$ is a vector space, $T$ is continuous t-representable and $P_{\mu, v}$ is a mapping $X \times(0,+\infty) \rightarrow L^{*}$ satisfying the following conditions for all $x, y \in X$ and $t$, $s>0$,

(a) $P_{\mu, v}(x, 0)=0_{L^{*}}$;

(b) $P_{\mu, v}(x, t)=1_{L^{*}}$ if and only if $x=0$;

(c) $P_{\mu, \nu}(\alpha x, t)=P_{\mu, v}(x, t / \alpha \mid)$ for all $a \neq 0$;

(d) $P_{\mu, v}(x+y, t+s) \geq_{L^{*}} T\left(P_{\mu, v}(x, t), P_{\mu, v}(y, s)\right)$.

Example 3.8 Let $(X,\|\cdot\|)$ be a normed space. Let $T(a, b)$ $=\left(a_{1} b_{1}, \min \left(a_{2}+b_{2}, 1\right)\right)$ for all $a=\left(a_{1}, a_{2}\right), b=\left(b_{1}, b_{2}\right) \in$ $L^{*}$ and let $\mu, v$ be measure and non-measure distribution functions defined by

$P_{\mu, v}(x, t)=\left(\mu_{x}(t), v_{x}(t)\right)=\left(\frac{t}{t+\|x\|}, \frac{\|x\|}{t+\|x\|}\right)$, for all $t \in \mathrm{R}^{+}$.

Then $\left(X, P_{\mu, v}, T\right)$ is an IRN-space.

Definition 3.9 (1) A sequence $\left\{x_{n}\right\}$ in an IRN-space $\left(X, P_{\mu, v}, T\right)$ is called a Cauchy sequence if, for any $\varepsilon>0$ and $t>0$, there exists an $n_{0} \in N$ such that $P_{\mu, v}\left(x_{n}-x_{m}, t\right)>_{L^{*}}\left(N_{s}(\varepsilon), \varepsilon\right) \quad$ for all $n, m \geq n_{0}$, where $N_{\mathrm{s}}$ is the standard negator.

(2) The sequence $\left\{x_{n}\right\}$ is said to be convergent to a point $x \in X$ (denoted by $x_{n} \stackrel{P_{\mu, v}}{\longrightarrow} x$ ) if $P_{\mu, v}\left(x_{n}-x, t\right) \rightarrow 1_{L^{*}}$ as $n \rightarrow \infty$ for every $t>0$.

(3) An IRN-space $\left(X, P_{\mu, v}, T\right)$ is said to be complete if every Cauchy sequence in $X$ is convergent to a point $x \in X$. 
Now, we prove the main result of this section as follows:

Theorem 3.10 Let $X$ be a linear space and $\left(Y, P_{\mu, v}, T\right)$ be a complete IRN-space. Let $f: X \rightarrow Y$ be a mapping with $f(0)$ $=0$ for which there exists $\xi, \zeta: X x X \rightarrow D^{+}$, where $\xi(x, y)$ is denoted by $\xi_{x, y}(t), \zeta(x, y)$ is denoted by $\zeta_{x, y}(t)$ and $\left(\xi_{x, y}(t), \zeta_{x, y}(t)\right)$ is denoted by $Q_{\xi, \zeta}(x, y, t)$ with the property $P_{\mu, v}(f(3 x+y)+f(x+3 y)-64 f(x)-64 f(y)-24 f(x+y)+6 f(x-y), t) \geq_{L^{\prime}} Q_{\xi, \zeta}(x, y, t)$

If $\quad T_{k=1}^{\infty}\left(Q_{\xi, \zeta}\left(3^{n+k-1} x, 0,3^{3 n+3 k+3} t\right)\right)=1_{L^{*}}$

$$
\lim _{n \rightarrow \infty} Q_{\xi, \zeta}\left(3^{n} x, 3^{n} y, 3^{n} t\right)=1_{L^{*}}
$$

for every $x, y \in X$ and $t>0$, then there exists a unique mapping $C: X \rightarrow Y$ such that

$P_{\mu, v}(f(x)-C(x), t) \geq_{L^{L}} T_{k=1}^{\infty}\left(Q_{\xi, \zeta}\left(3^{k-1} x, 0,3^{3 k+3} t\right)\right)$

Proof: Let $0<\alpha<3^{4}$ and $y=0$ in (3.1), we obtain

$$
\begin{aligned}
& P_{\mu, v}(f(3 x)+f(x)-64 f(x)-64 f(0)-24 f(x)+6 f(x), t) \geq_{L^{*}} Q_{\xi, \zeta}(x, 0, t) \\
& P_{\mu, v}\left(f(3 x)-3^{4} f(x), t\right) \geq_{L^{*}} Q_{\xi, \zeta}(x, 0, t) \\
& P_{\mu, v}\left(\frac{f(3 x)}{3^{4}}-f(x), \frac{t}{3^{4}}\right) \geq_{L^{*}} Q_{\xi, \zeta}\left(x, 0,3^{4} t\right)
\end{aligned}
$$

Therefore, it follows that replacing $x$ with $3^{k} x$, we have

$$
P_{\mu, v}\left(\frac{f\left(3^{k+1} x\right)}{3^{4(k+1)}}-\frac{f\left(3^{k} x\right)}{3^{4 k}}, \frac{t}{3^{4 k}}\right) \geq_{L^{*}} Q_{\xi, \zeta}\left(3^{k} x, 0,3^{4} t\right)
$$

which shows that

$$
P_{\mu, v}\left(\frac{f\left(3^{k+1} x\right)}{3^{4(k+1)}}-\frac{f\left(3^{k} x\right)}{3^{4 k}}, t\right) \geq_{L^{*}} Q_{\xi, \zeta}\left(3^{k} x, 0,3^{4(k+1)} t\right)
$$

that is

$$
P_{\mu, v}\left(\frac{f\left(3^{k+1} x\right)}{3^{4(k+1)}}-\frac{f\left(3^{k} x\right)}{3^{4 k}}, \frac{t}{3^{(k+1)}}\right) \geq_{L} Q_{\xi, \zeta}\left(3^{k} x, 0,3^{3(k+1)} t\right)
$$

for all $k \in N$ and $t>0$. As $1>\frac{1}{3}+\frac{1}{3^{2}}+\frac{1}{3^{3}}+\ldots . \frac{1}{3^{n}}$ with the single inequality, it follows that

$$
\begin{aligned}
& P_{\mu, v}\left(\frac{f\left(3^{n} x\right)}{3^{4 n}}-f(x), t\right) \geq_{L^{*}} P_{\mu, v}\left(\frac{f\left(3^{n} x\right)}{3^{4 n}}-f(x), \sum_{k=0}^{n-1} \frac{1}{3^{k+1}} t\right) \\
& \geq_{L^{*}} T_{k=0}^{n-1}\left(P_{\mu, v}\left(\frac{f\left(3^{k+1} x\right)}{3^{4(k+1)}}-\frac{f\left(3^{k} x\right)}{3^{4 k}}, \frac{t}{3^{k+1}}\right)\right) \\
& \geq_{L^{*}} T_{k=0}^{n-1}\left(Q_{\mu, v}\left(3^{k-1} x, 0,3^{3 k+3} t\right)\right)
\end{aligned}
$$

To prove the convergence of the sequence $\left\{\frac{f\left(3^{n} x\right)}{3^{4 n}}\right\}$, replacing $x$ with $3^{m} x$ in the above inequality (3.9), we get

$$
\begin{aligned}
& P_{\mu, v}\left(\frac{f\left(3^{n+m} x\right)}{3^{4(n+m)}}-\frac{f\left(3^{m} x\right)}{3^{4 m}}, t\right) \\
& \geq_{L^{*}} T_{k=0}^{n-1}\left(Q_{\mu, v}\left(3^{k+m-1} x, 0,3^{3 k+3 m+3} t\right)\right)
\end{aligned}
$$

since the right hand side of the inequality tends to $1_{L^{*}}$ as $\mathrm{m}$ tends to $\infty$, hence the sequence $\left\{\frac{f\left(3^{n} x\right)}{3^{4 n}}\right\}$ is a Cauchy sequence since $Y$ is a complete IRN-space. Therefore, we may define $C(x)=\lim _{n \rightarrow \infty} \frac{f\left(3^{n} x\right)}{3^{4 n}}$, for all $x$ in $X$.

On Replacing $x$ and $y$ with $3^{n} x$ and $3^{n} y$ respectively in (3.1) and taking the limit as $n \rightarrow \infty$, we find that $C$ is a quartic mapping for all $x, y \in X$ and taking limit as $n \rightarrow \infty$, inequality (3.9) implies (3.4).

To prove the uniqueness of the quartic mapping $C$, let us assume that there exists another cubic function $C^{1}$ which satisfies (3.4). Obviously, we have $C\left(3^{n} x\right)=3^{4 n} C(x)$ and $C^{1}\left(3^{n} x\right)=3^{4 n} C^{1}(x)$ for all $x \in X$ and $n \in N$. Hence, it follows from (3.2) and (3.4) that

$$
\begin{gathered}
P_{\mu, v}\left(C(x)-C^{1}(x), t\right) \geq_{L^{*}} P_{\mu, v}\left(C\left(3^{n} x\right)-C^{1}\left(3^{n} x\right), 3^{4 n} t\right) \\
P_{\mu, v}\left(C(x)-C^{1}(x), t\right) \geq_{L^{*}} \\
T_{\mu, v}\left(P_{\mu, v}\left(C\left(3^{n} x\right)-f\left(3^{n} x\right), 3^{4 n-1} t\right), P_{\mu, v}\left(f\left(3^{n} x\right)-C^{1}\left(3^{n} x\right), 3^{4 n-1} t\right)\right) \\
P_{\mu, v}\left(C(x)-C^{1}(x), t\right) \geq_{L^{*}} \\
T\left(T_{k=1}^{\infty}\left(Q_{\xi, \zeta}\left(3^{n+k-1} x\right), 0,3^{4 n+3 k+1} t\right), T_{k=1}^{\infty}\left(Q_{\xi, \zeta}\left(3^{n+k-1} x\right), 0,3^{4 n+3 k+3} t\right)\right) \\
=T\left(1_{L^{*}}, 1_{L^{*}}\right)=1_{L^{*}}
\end{gathered}
$$

for all $x$ in $X$. Hence the desired result.

Corollary 3.11 Let $\left(X, P_{\mu^{1}, v^{1}}^{1}, T\right)$ be an IRN-space and $\left(Y, P_{\mu, v}, T\right)$ be a complete IRN-space. Let $f: X \rightarrow Y$ be a mapping such that for $t>0$,

$P_{\mu, v}(f(3 x+y)+f(x+3 y)-64 f(x)-64 f(y)-24 f(x+y)+6 f(x-y)) \geq_{L^{L}} P_{\mu^{\prime}, y^{\prime}}^{1}(x+y, t)$ where $\lim _{n \rightarrow \infty} T_{k=1}^{\infty}\left(P_{\mu^{1}, v^{1}}^{1}\left(3^{n+k-1} x, 3^{3 n+3 k+3} t\right)\right)=1_{L^{L}}$ for all $x, y \in X$.

Then there exists a unique quartic mapping $C: X \rightarrow Y$ such that $P_{\mu, v}(f(x)-C(x), t) \geq_{L^{*}} T_{k=1}^{\infty}\left(P_{\mu^{1}, v^{1}}^{1}\left(3^{k-1} x, 3^{3 k+3} t\right)\right)$

Example 3.12 Let $(X,\|\|$.$) be a Banach algebra space,$ $\left(X, P_{\mu, v}, T\right)$ be an IRN-space in which

$$
P_{\mu, v}(x, t)=\left(\frac{t}{t+\|x\|}, \frac{\|x\|}{t+\|x\|}\right)
$$


and $\left(Y, P_{\mu, v}, T\right)$ be a complete IRN-space for all $x$ in $X$.

Define $f: X \rightarrow Y$ by $f(x)=x^{4}+x_{0}$ where $x_{0}$ is a unit element in $X$. Therefore for all $t>0$

$$
\begin{aligned}
P_{\mu, v}(f(3 x+y)+ & f(x+3 y)-64 f(x)-64 f(y)-24 f(x+y)+6 f(x-y), t) \geq_{L^{*}} P_{\mu, v}(x+y, t) \\
& \lim _{n \rightarrow \infty} M_{k=1}^{\infty}\left(P_{\mu, v}\left(3^{n+k-1} x, 3^{3 n+3 k+3} y\right)=\right. \\
& \lim _{n \rightarrow \infty} \lim _{n \rightarrow \infty} M_{k=1}^{\infty}\left(P_{\mu, v}\left(x, 3^{2 k+2 n+3} t\right)\right) \\
= & \lim _{n \rightarrow \infty} \lim _{n \rightarrow \infty}\left(P_{\mu, v}\left(x, 3^{2 n+4} t\right)\right)=1_{L^{*}}
\end{aligned}
$$

Therefore, all the conditions of theorem 3.10 holds and there exists a unique quartic mapping $C: X \rightarrow Y$ such that $P_{\mu, v}(f(x)-C(x), t) \geq_{L^{*}} P_{\mu, v}\left(x, 3^{3} t\right)$.

\section{CONCLUSION}

In this paper, we concluded the following results:

1. In section 2, we studied the Hyers-Ulam-Rassias stability of the quartic functional equation (1.1) using fixed point method on Random normed spaces.

2. The Hyers-Ulam-Rassias stability of the Quartic functional equation (1.1) on intuitionistic random normed space is studied in section 3 .

3. We also present some corollaries and an examples related to our results.

\section{ACKNOWLEDGMENTS}

This Research is supported by the University Grant Commission of India (Grant No. 39-29/2010(SR))

\section{REFERENCES}

[1] A. N. Sherstnev : On the notion of a random normed space. Dpkl. Akad. Nauk SSSR 149 (1963), pp. 280283 (in Russian).

[2] B. Schweizer and A. Sklar, Probabilistic Metric Spaces. Elsevier, North Holand (1983).

[3] C. Jung, On generalized complete metric spaces. Bull Am Math Soc. 75 (1969), pp. 113-116.

[4] D. H. Hyers, On the Stability of the Linear Functional Equation, Proc. Nat. Acad. Sci. U.S.A. 27 (1941), pp. 222-224.
[5] D. Miheţ and V. Radu, On the stability of the additive Cauchy functional equation in random normed spaces. J Math Anal. Appl. 343(2008), pp. $567-572$.

[6] D. Miheț, The stability of the additive Cauchy functional equation in non-Archimedean fuzzy normed spaces. Fuzzy Set Syst. 161 (2010), pp. 2206-2212.

[7] G. Deschrijver, and EE. Kerre, On the relationship between some extensions of fuzzy set theory. Fuzzy Set Syst. 23 (2003), pp. 227-235.

[8] O. Hadžić and E. Pap, Fixed Point Theory in PMSpaces. Kluwer Academic, Dordrecht (2001)

[9] K. Atanassov, Intuitionistic fuzzy sets. Fuzzy Set Syst. 20 (1986), pp. 87-96.

[10] M. Petapirak and P. Nakmahachalasiant, A quartic functional equation and it Generalized Hyers-UlamRassias stability, Thai J. of Mat., Sp. Iss.(2008) pp.77-84.

[11] P. Gavruta : A Generalization of the Hyers-UlamRassias Stability of Approximately Additive Mappings, J. Math. Anal. Appl. 184 (1994), pp.431436.

[12] R. Saadati, J. Park, On the intuitionistic fuzzy topological spaces, Chaos Soliton Fract. 27(2006), pp. 331-344.

[13] S. Chang, J. Rassias and R. Saadati, The stability of the cubic functional equation in intuitionistic random normed spaces, Appl Math Mech. 31 (2010), pp. 2126.

[14] S. Chang, Y. Cho and Y. Kang, Nonlinear Operator Theory in Probabilistic Metric Spaces. Nova Science Publishers Inc., NewYork (2001)

[15] S. Kutukcu, A. Tuna and A. Yakut, Generalized contraction mapping principle in intuitionistic Menger spaces and application to differential equations. Appl Math Mech. 28 (2007) pp.799-809.

[16] S.M. Ulam : Problems on Modern Mathematics, John Wiley and Sons, New York, NY, USA, 1964.

[17] Th. M. Rassias, On the stability of the Linear mapping in Banach spaces, Procc. Of the American Mathematical Society, vol. 72(1978), no. 2, pp.297300 . 